\title{
Mid-infrared interferometry of massive young stellar objects
}

\section{VLTI and Subaru observations of the enigmatic object M8E-IR ${ }^{\star}$}

\author{
H. Linz ${ }^{1}$, Th. Henning ${ }^{1}$, M. Feldt ${ }^{1}$, I. Pascucci ${ }^{2}$, R. van Boekel ${ }^{1}$, A. Men'shchikov ${ }^{3}$, B. Stecklum ${ }^{4}$, O. Chesneau $^{5}$, \\ Th. Ratzka ${ }^{6}$, S. P. Quanz ${ }^{1}$, Ch. Leinert ${ }^{1}$, L. B. F. M. Waters ${ }^{7}$, and H. Zinnecker ${ }^{6}$ \\ 1 Max-Planck-Institut für Astronomie, Königstuhl 17, 69117 Heidelberg, Germany \\ e-mail: [linz; henning; feldt; leinert; boekel; quanz] @mpia-hd.mpg.de \\ 2 Department of Physics and Astronomy, Johns Hopkins University, 403 Bloomberg Center, 3400 N. Charles Street, Baltimore, \\ MD 21218, USA \\ e-mail: pascucci@pha.jhu.edu \\ 3 CEA, IRFU, SAp, Centre de Saclay, 91191 Gif-sur-Yvette, France \\ e-mail: alexander.menshchikov@cea.fr \\ 4 Thüringer Landessternwarte Tautenburg, Sternwarte 5, 07778 Tautenburg, Germany \\ e-mail: stecklum@tls-tautenburg.de \\ 5 UMR 6525, Univ. Nice, CNRS, Obs. de la Côte d'Azur, Av. Copernic, 06130 Grasse, France \\ e-mail: chesneau@obs-azur.fr \\ 6 Astrophysikalisches Institut Potsdam, Sternwarte 16, 14482 Potsdam, Germany \\ e-mail: [tratzka; hzinnecker]@aip.de \\ 7 Astronomical Institute "Anton Pannekoek", Universiteit van Amsterdam, Kruislaan 403, 1098 SJ Amsterdam, The Netherlands \\ e-mail: rensw@science.uva.nl
}

Received 30 November 2007 / Accepted 2 July 2009

\section{ABSTRACT}

\begin{abstract}
Context. Our knowledge of the inner structure of embedded massive young stellar objects is still quite limited. Thus, it is difficult to decide to what extent the mass accumulation onto forming massive stars differs from the process of low-mass star formation. Aims. We attempt to overcome the spatial resolution limitations of conventional thermal infrared imaging.

Methods. We employed mid-infrared interferometry using the MIDI instrument on the ESO/VLTI facility to investigate M8E-IR, a well-known massive young stellar object suspected of containing a circumstellar disk. Spectrally dispersed visibilities in the $8-13 \mu \mathrm{m}$ range have been obtained at seven interferometric baselines.

Results. We resolve the mid-infrared emission of M8E-IR and find typical sizes of the emission regions of the order of 30 milliarcseconds ( $=45 \mathrm{AU}$ ). Radiative transfer simulations were performed to interpret the data. The fitting of the spectral energy distribution, in combination with the measured visibilities, does not provide evidence for an extended circumstellar disk with sizes $\gtrsim 100$ AU but requires the presence of an extended envelope. The data are not able to constrain the presence of a small-scale disk in addition to an envelope. In either case, the interferometry measurements indicate the existence of a strongly bloated, relatively cool central object, possibly tracing the recent accretion history of M8E-IR. In addition, we present $24.5 \mu$ m images that clearly distinguish between M8EIR and the neighbouring ultracompact HII region and which show the cometary-shaped infrared morphology of the latter source. Conclusions. Our results show that IR interferometry, combined with radiative transfer modelling, can be a viable tool to reveal crucial structure information on embedded massive young stellar objects and to resolve ambiguities arising from fitting the SED.
\end{abstract}

Key words. stars: formation - techniques: interferometric - radiative transfer - stars: individual: M8E-IR

\section{Introduction}

High-mass stars predominantly form in clustered environments much farther away from the Sun, on average, than typical wellinvestigated low-mass star-forming regions. Thus, high spatial resolution is a prerequisite for making progress in the observational study of high-mass star formation. Furthermore, all phases prior to the main sequence are obscured by dense circumstellar environments. This forces observers of deeply embedded massive young stellar objects (MYSOs) to move to the mid-infrared (MIR), where the resolution of conventional imaging is limited to $>0$ '. 25 even with $8-\mathrm{m}$ class telescopes. Hence, one traces linear scales still several hundred AU in size even for the nearest

* Based on observations within the ESO programs 073.C-0175(A), 273.C-5044(A), 074.C-0389(B), and 075.C-0755(A, B).
MYSOs, and conclusions on the geometry of the innermost circumstellar material remain ambiguous. MIR emission moderately resolved with single-dish telescopes could even arise from the inner outflow cones (e.g., De Buizer 2006; Linz et al. 2005). A way to overcome the diffraction limit of single telescopes is to employ interferometric techniques. We are presently conducting a larger survey toward MYSOs based on MIR interferometry. While the results for the other objects will be reported in subsequent publications, we concentrate here on the object M8E-IR, a prominent BN-type MYSO (cf. Henning 1990) at a distance of 1.25-1.5 kpc (Arias et al. 2007; Simon et al. 1984). Although M8E-IR had been well investigated in the 1980s, the spatial resolution for most of the IR observations of M8E-IR was poor. An exception is the work by Simon et al. (1985) who speculated on the existence of a small circumstellar disk around M8E-IR based 
Table 1. Log of MIDI observations of M8E-IR.

\begin{tabular}{llcccc}
\hline \hline UT date and time & $\begin{array}{c}B \\
{[\mathrm{~m}]}\end{array}$ & $\begin{array}{c}\text { PA } \\
{[\mathrm{deg}]}\end{array}$ & $\begin{array}{c}\text { Telescope } \\
\text { pair }\end{array}$ & $\begin{array}{c}\text { ESO } \\
\text { Program }\end{array}$ \\
\hline $2004-06-05$ & $08: 07$ & 96.8 & +42.7 & U1-U3 & $073 . C-0175(\mathrm{~A})$ \\
$2004-06-05$ & $09: 57$ & 82.2 & +44.5 & U1-U3 & $073 . C-0175(\mathrm{~A})$ \\
$2004-08-01$ & $01: 51$ & 46.6 & +38.4 & U2-U3 & 273.C-5044(A) \\
$2005-03-02$ & $08: 58$ & 46.8 & -85.9 & U3-U4 & $074 . C-0389(\mathrm{~B})$ \\
$2005-06-24$ & $07: 37$ & 51.6 & -42.2 & U3-U4 & $075 . C-0755(\mathrm{~B})$ \\
$2005-06-24$ & $09: 29$ & 43.4 & -10.6 & U3-U4 & $075 . C-0755(\mathrm{~B})$ \\
$2005-06-26$ & $00: 26$ & 55.7 & -06.6 & U1-U2 & $075 . C-0755(\mathrm{~A})$ \\
\hline
\end{tabular}

on thermal infrared lunar occultation data. Here, we present our work on M8E-IR which includes 8-13 $\mu \mathrm{m}$ interferometry to dissect the MYSO M8E-IR itself, and $N$ - and $Q$-band imaging to have a fresh look at M8E-IR in its relation to the environment.

\section{Observations}

\subsection{Mid-infrared imaging with COMICS at Subaru}

The SMOKA data archive (Baba et al. 2002) contains 8.2-m Subaru observations of M8E-IR in the thermal infrared, obtained on June 08, 2004 with the camera COMICS (Okamoto et al. 2003), utilising the $24.5 \mu \mathrm{m} Q$-band filter $\left(\lambda_{\mathrm{c}}=24.56 \mu \mathrm{m}\right.$, $\Delta \lambda=0.75 \mu \mathrm{m})$ as well as the $10.5 \mu \mathrm{m} \mathrm{N}$-band filter $\left(\lambda_{\mathrm{c}}=\right.$ $10.48 \mu \mathrm{m}, \Delta \lambda=1.05 \mu \mathrm{m})$. For imaging, COMICS employs one $320 \times 240$ pixel $^{2} \mathrm{Si}$ :As IBC detector from Raytheon with a nominal pixel scale of 0.13 arcsec. For the observations, the whole detector was read out with an elementary exposure time of $0.06 \mathrm{~s}$. These exposures were repeated 16 times per chop position. Chopping with a frequency of $1 \mathrm{~Hz}$, a nominal throw of $20^{\prime \prime}$ and a chop angle of $140^{\circ}$ was applied to remove the strong thermal background. Since for COMICS/Subaru, the residual pattern after chopping subtraction is negligible for most purposes (cf. Fujiwara et al. 2006), additional nodding was not applied during the observations. The total integration time (chop-on plus chop-off) is $400 \mathrm{~s}$ in $Q$-band and $200 \mathrm{~s}$ in $N$ band, respectively. The stars HD 148478 ( $Q$-band) and HD 169916 ( $N$-band) were observed directly before and after M8E-IR for flux calibration.

\subsection{MIDI interferometry at the VLTI}

Visibilities in the mid-infrared wavelength range $8-13 \mu \mathrm{m}$ have been obtained with the instrument MIDI (Leinert et al. 2003) at the Very Large Telescope Interferometer. Within the framework of Guaranteed Time Observations for MIDI as well as Director's Discretionary Time, we observed M8E-IR at seven baseline length/baseline orientation combinations between June 2004 and June 2005. In Table 1 we list the UT dates and UT times for the fringe track data, the projected baseline lengths and the position angles of the projected baselines on the sky (counted from north via east on the sky), as well as the telescope configurations used and the observing proposal numbers. We refer to Leinert et al. (2004) for a more detailed description of the standard observing procedure for MIDI observations. For all our observations, the so-called HighSens mode was used: during self-fringe tracking, all the incoming thermal infrared signal is used for beam combination and fringe tracking, while the photometry is subsequently obtained in separate observations. We use the MIDI prism as the dispersing element, hence, we finally obtain spectrally dispersed visibilities with a spectral resolution of $R \approx 30$. HD 169916 was used as the main interferometric and photometric standard star and always was observed immediately after M8E-IR. In addition, all calibrator measurements of a night were collected to create an average interferometric transfer function and to assign error margins to the measured visibilities. For the August 01, 2004 observations, the conditions were almost photometric, the airmass of both M8E-IR and HD 169916 was minimal (1.01), and the data are hardly affected by atmospheric disturbances (e.g., ozone feature). Therefore, we use the dispersed photometry from this measurement to provide the $\mathrm{N}$-band spectrum later used in the SED fitting (see Sect. 3.3).

\section{Results}

\subsection{MIR imaging}

In Fig. 1, we show the $24.5 \mu \mathrm{m}$ Subaru/COMICS image on the left. M8E-IR is still the dominating source at this wavelength. At a nominal resolution of 0.75 , the emission remains compact and barely differs from the profile of the supposedly unresolved calibrator star. In a very recent MIR survey paper by de Wit et al. (2009), the same Subaru data set is used to analyse the $24.5 \mu \mathrm{m}$ intensity profile of M8E-IR in more detail and to model the profile and the SED by means of spherically symmetric model configurations. These authors come to the same conclusion regarding the compactness of the M8E-IR profile. A second faint point source not yet reported in the literature is visible roughly 6 " west of it. Furthermore, we clearly detect MIR emission arising from the neighbouring radio source (Simon et al. 1984; Molinari et al. 1998). Simon et al. (1985) had already reported a detection of this source in $N$ - and $Q$-band (fluxes only, measured with a 6" diaphragm). For the first time, the Subaru MIR imaging spatially resolves this emission. It is comet-shaped, with the apex directed away from M8E-IR. This morphology could be an intrinsic property of this UCHII region, or it could be shaped by the molecular outflow probably arising from M8E-IR (Mitchell et al. 1988, 1992) onto this radio source. Damiani et al. (2006) reported an X-ray source $\approx 1$ 1.'7 southwest of M8E-IR ([DFM2004] 845). Accounting for the uncertainty of the X-ray position (2".56), the $\mathrm{X}$-ray emission can still be associated with M8E-IR, but not with the known radio source or the abovementioned faint MIR point source.

At $10.5 \mu \mathrm{m}$ we easily detect M8E-IR (Fig. 1, right). The source is very bright, which causes some additional image artefacts (diffraction spikes in diagonal directions as well as a detector "drooping" effect, causing multiple fainter copies of the strong source along the north-south direction). Hence, we will not further investigate the shape of M8E-IR in this image. The radio source counterpart is not detected at $10.5 \mu \mathrm{m}(\mathrm{rms}$ noise $\approx 8 \mathrm{mJy}$ ). Considering the COMICS filter characteristics (Sect. 2.1), combined with the detection of the radio counterpart by Simon et al. (1985) in a broad $N$-band filter (0.97 Jy), this implies a very strong and broad $9.7 \mu \mathrm{m}$ silicate absorption feature or time variability. The third source in the $24.5 \mu \mathrm{m}$ image is detected at a $4 \sigma$ level at $10.5 \mu \mathrm{m}$.

\subsection{MIR interferometry}

We reduced the interferometric data with the MIA+EWS package, version 1.5, developed at the MPIA Heidelberg and the University of Leiden. The resulting visibility curves are collected in Fig. 2, left. The object is clearly resolved in all configurations with visibilities between $0.09-0.35$. If we assume a 


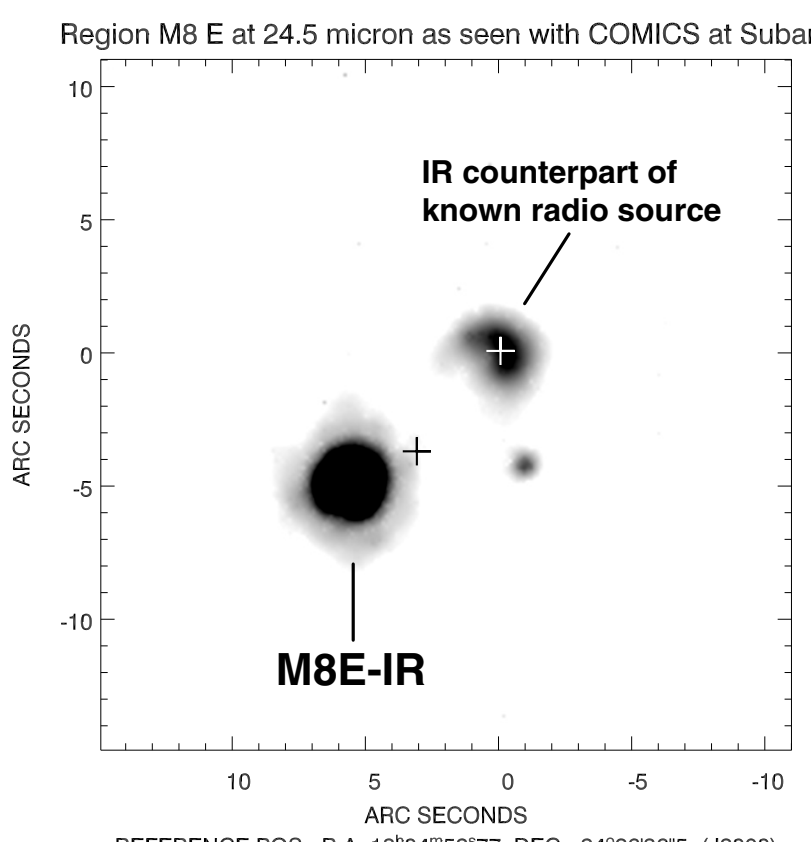

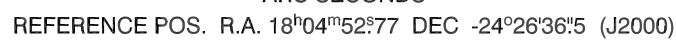

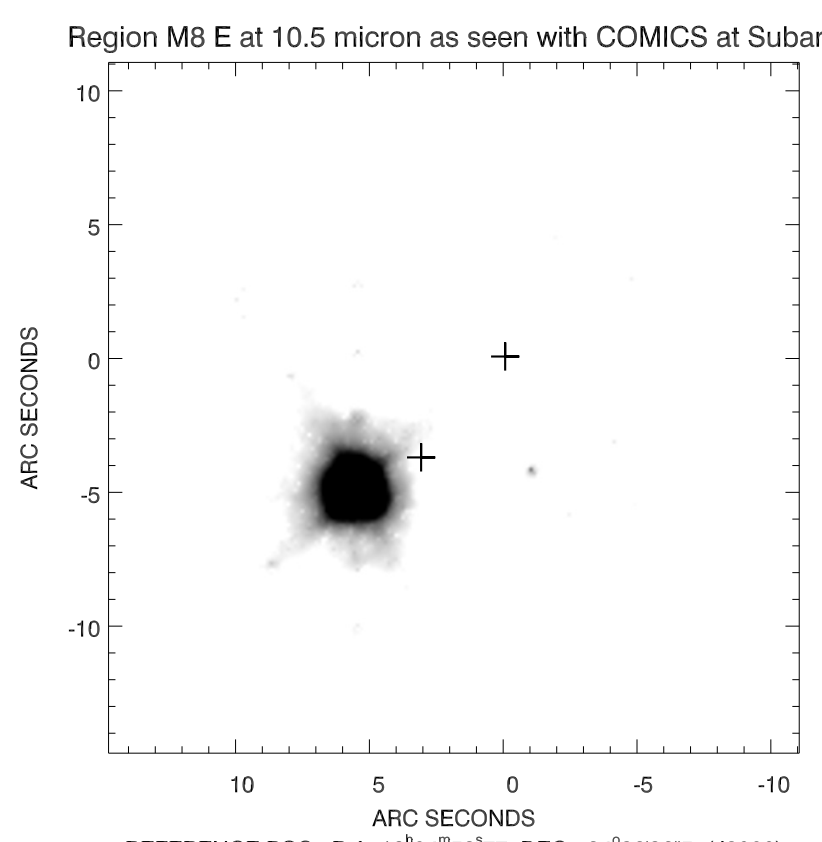

REFERENCE POS. R.A. $18^{\mathrm{h}} 04^{\mathrm{m}} 52^{\mathrm{s}} .77$ DEC $-24^{\circ} 26^{\prime} 36 . " 5$ (J2000)

Fig. 1. Left: $Q$-band image of the M8E region at $24.5 \mu \mathrm{m}$ with COMICS/Subaru. The two previously known objects are annotated. The black plus indicates the position of a $44 \mathrm{GHz}$ methanol maser according to Val'tts (1999). The white plus marks the position of the $\mathrm{cm}$ continuum source (Molinari et al. 1998). Right: $N$-band image of the M8E region at $10.5 \mu \mathrm{m}$ with COMICS/Subaru. The two plus signs mark the same objects as in the left image. The IR counterpart of the $\mathrm{cm}$ source is not detected, while the third IR source is faintly visible.

MIDI Visibilities for M8E-IR

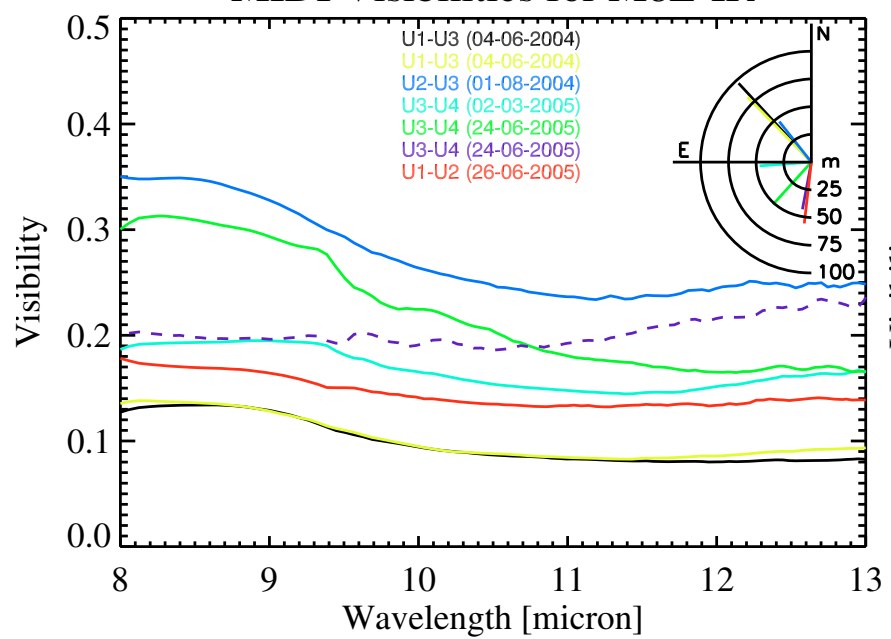

47-m baseline comparison

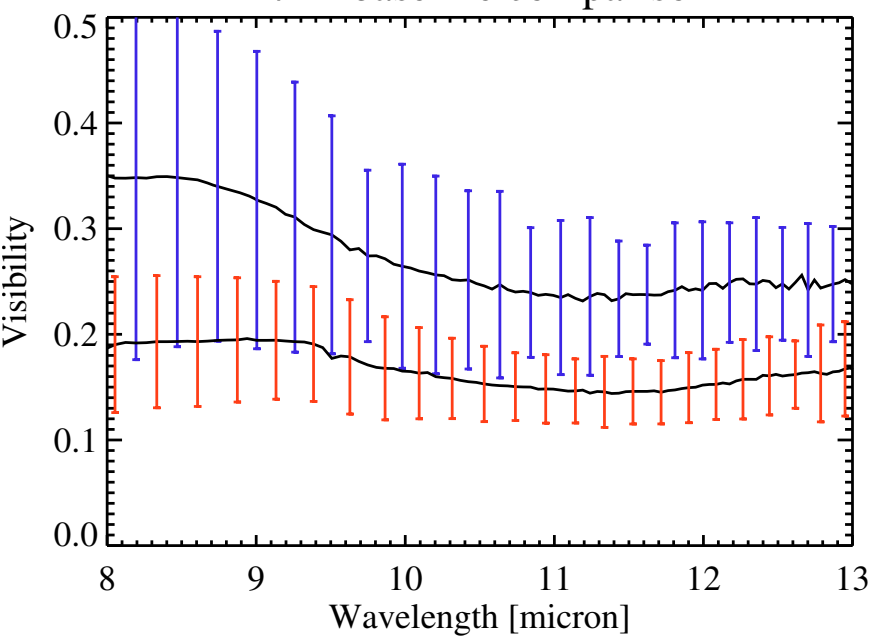

Fig. 2. Left: collection of dispersed MIDI visibilities. The inset schematically shows the baseline lengths and orientations (cf. Table 1). Note that the apparently rising slope of the purple UT3-UT4 visibility curve from 24-06-2005 (dashed line) is probably an artefact of the measurement at very high airmass (see Sect. 3.2). Right: the two visibility curves with very similar projected baseline lengths (46.6 m vs. $46.8 \mathrm{~m})$ but strongly differing position angles $\left(+38.4^{\circ}\right.$, upper curve vs. $-85.9^{\circ}$, lower curve). The formal error bars $(+/-3 \sigma)$ are indicated.

Gaussian intensity distribution ${ }^{1}$ of the source, the visibilities indicate an intensity $F W H M$ of $\approx 20-25$ mas $(8.5 \mu \mathrm{m})$ and $32-$ $38 \mathrm{mas}(12.0 \mu \mathrm{m})$ which is in rough agreement with the extension of the small component of Simon et al. (1985). We note that these visibilities, although not reaching the relatively high levels of most Herbig Ae/Be stars (e.g., Leinert et al. 2004), are qualitatively different from the very low visibilities $(0.01-0.05)$ found for several of the other objects in our sample as well as recently

\footnotetext{
1 The relation between the visibility and the intensity full width half maximum is given by $V(u)=\exp \left(-\pi^{2} /(4 \ln 2) u^{2} \mathrm{FWHM}^{2}\right)$ in this case, where the spatial frequency is $u=\lambda / B$, with $B$ being the modulus of the projected baseline length on the sky.
}

reported for two other massive YSOs (de Wit et al. 2007; Vehoff et al. 2008). Still, to learn more about the potentially more complicated intensity structure, a simple Gaussian is not a sufficient ansatz, especially if the object is strongly resolved by the interferometer. Further modelling is necessary for interpretation.

Can we infer the geometry of the source from our visibility curves alone? Most visibilities have been taken at different baseline length/position angle combinations. Still, there are two measurements with almost the same projected baseline length (46.6 m vs. $46.8 \mathrm{~m}$ ), but position angles differing by $55.7^{\circ}$ (cf. Table 1). A spherically symmetric intensity distribution will result in visibilities not being a function of position angle. These two measurements, however, show different visibility 
levels (Fig. 2, right). But when considering the 3- $\sigma$ error bars, this difference is not fully conclusive, since the error bars, at least in the 8 to $11 \mu \mathrm{m}$ range, still overlap slightly. At longer wavelengths, the two error intervals are almost distinct. While we cannot draw strong conclusions from these findings, this is a first hint that the mid-infrared intensity distribution of M8E-IR is indeed not fully spherically symmetric.

Visibilities can be considered as the ratio of correlated flux to total flux. The $N$ band total flux spectrum of M8E-IR shows a moderately deep silicate absorption spectrum (see inset of Fig. 3). Most of the curves displayed in Fig. 2 (left) show a drop in the visibility level over the silicate feature. However, the visibility curve corresponding to the 2005-06-24 09:29 UT measurement does not show this behaviour, but instead rises monotonically from 8 to $13 \mu \mathrm{m}$. We think that this is an artefact of the observation. While all the other measurements were taken at intermediate elevations (airmass $<1.73$ ), the data set in question was obtained at a very low elevation (airmass 2.41) in order to get this $(u, v)$ point, corresponding to a relatively short projected baseline length. At such a low elevation, the performance of MIDI is certainly affected. Since the adaptive optics system cannot be locked on M8E-IR itself, but only on a faint optical star more than $40^{\prime \prime}$ away, the beam stability and therefore the beam overlap on the MIDI detector may be compromised. Since the PSF is smaller at 8 micron than at 13 micron, a smaller beam overlap will lead to apparent losses in correlated flux preferentially on the short-wave side of the spectrum. We have critically checked the overlap for this measurement and indeed find that the two beams are more separated than for other observations of M8E-IR as well as for the calibrators. By artificially restricting the overlap mask to a smaller central area, the slope of the visibility curve can be flattened with (calibrated) visibility values at 8 micron slightly higher than at 13 micron. Hence, the low 8 micron correlated flux of the indicated visibility curve is an artefact of the measurement process. Therefore, we will not further interpret the shape of this particular visibility curve.

\subsection{Modelling}

We apply self-consistent continuum radiative transfer modelling to M8E-IR in order to produce synthetic MIR intensity maps and to compare their spatial frequency spectrum with the observed visibilities. Self-consistency here means that the dust temperature is calculated from the condition of (radiative) energy conservation. Hence, the luminosity sources in the system (central object + accretion) initially heat up the dust until a radiative equilibrium is reached, the temperature (for the given dust density structure) converges, and the total luminosity is recovered in the emerging SED (Bjorkman \& Wood 2001; Whitney et al. 2003; Men'shchikov \& Henning 1997). We are mainly concerned with the question of which spatial distribution of the circumstellar material can account for both the SED and the visibilities of M8E-IR.

For SED fitting, we use the M8E-IR photometric data collected in Mueller et al. (2002) plus new $1.2 \mathrm{~mm}$ data from Beltrán et al. (2006). We stress that no (sub-)millimeter interferometry on M8E-IR is reported in the literature which could spatially disentangle the flux contributions from M8E-IR and the radio source $8^{\prime \prime}$ away. Hence, we consider the fluxes for $\lambda>24.5 \mu \mathrm{m}$ as upper limits in our modelling. Furthermore, we include new optical photometry in the $B, V$, and $I$ filters reported by Prisinzano et al. (2005, their object $\mathrm{Cl}^{*} \mathrm{NGC}$ 6530 WFI 13458) as well as our new $24.5 \mu \mathrm{m}$ photometry $(220 \pm 20 \mathrm{Jy})$. The $8-13 \mu \mathrm{m}$ total flux spectrum taken during

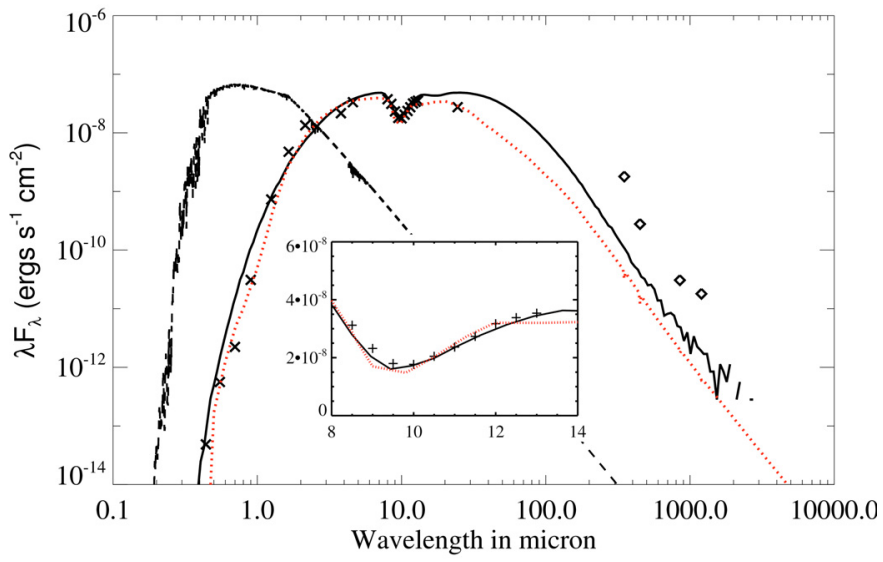

Fig. 3. SED of M8E-IR. Crosses = observed fluxes, diamonds $=$ upper limits. The solid line gives the best-fitting radiative transfer model 3007526 from the grid of models by Robitaille et al. (2007) (effective synthetic aperture $\sim 2^{\prime \prime}$ ). The dashed black curve shows the SED of the bloated central star used in that model. The red dotted line shows the spherical model with a hot star (also mentioned in Sect. 3.3) that fits the SED but not the visibilities (cf. Fig. 5). The inset shows a zoom into the $10 \mu \mathrm{m}$ region using a synthetic aperture $<1^{\prime \prime}$, appropriate for the underlying MIDI spectroscopy data (shown as plus signs). For legibility, the symbol sizes are similar to or larger than the real error bars.

Table 2. Parameters of the best-fitting Robitaille model 3007526.

\begin{tabular}{lrlr}
\hline \hline Parameter & Value & Parameter & Value \\
\hline Effect. temperature & $4,740 \mathrm{~K}$ & Stellar radius & $125 R_{\odot}$ \\
Stellar mass & $13.5 \mathrm{M}_{\odot}$ & Disk mass & $0.71 M_{\odot}$ \\
Outer disk radius $^{(1)}$ & $16 \mathrm{AU}$ & Density exp. $^{(1)}$ & 2.05 \\
Scale height exp. $^{(1)}$ & 1.05 & ${\text { Viscosity } \alpha \text { par. }^{(1)}}^{(2)}$ & $1.3 \mathrm{E}-2$ \\
Envelope radius $^{\text {tot }}$ & $6.8 \mathrm{E}+4 \mathrm{AU}$ & Mass infall rate $^{(2)}$ & $7 \mathrm{E}-5 M_{\odot} / \mathrm{yr}$ \\
$A_{\mathrm{V}}$ along LOS & $33 \mathrm{mag}$ & Inclination angle $^{\circ}$ & $18^{\circ}$ \\
Cavity cone angle & $3.3^{\circ}$ & & \\
\hline
\end{tabular}

${ }^{(1)}$ For the disk; ${ }^{(2)}$ for the envelope; dust model: Kim et al. (1994).

the MIDI measurements (Sect. 2.2) was used to further curtail the number of viable models.

We used the SED online fitting tool of Robitaille et al. (2007) that can in principle utilise models including an envelope plus circumstellar disk. We refer to this publication for details on the setup of these models. To produce the synthetic MIR intensity maps corresponding to the Robitaille models, we use the underlying Monte Carlo radiative transfer code of Whitney et al. (2003). The SED fitting tool delivers the full parameter set for the best-fitting models which can be directly included in the Whitney code. The resulting images are then Fouriertransformed, and cuts through this spatial frequency spectrum of the images give the synthetic visibilities.

Intriguingly, we do not find a unique solution from this SED fitting. The formally best SED fit points to a model featuring a very compact circumstellar disk ( $<20 \mathrm{AU})$, a larger surrounding envelope with small bipolar cavities and a bloated cooler central object (cf. Table 2). In Fig. 3, we show this best SED fit to M8E-IR by the Robitaille models as black continuous line. Due to the linkage of the Robitaille grid to evolutionary tracks, certain size parameters cannot be independently chosen by the fitter (Robitaille 2008). This especially affects the possible disk size which is not well constrained in our case. The data would still allow for a somewhat larger disk. We have repeated the calculation of the intensity maps with all model parameters fixed to 
the values of the model best fitting the SED, but have used larger disk sizes of 50, 100, 250, and 500 AU. For disk radii $\gtrsim 100 \mathrm{AU}$, the resulting MIR visibilities eventually drop. Hence, the difference to the values measured with MIDI increases, which makes such solutions less likely.

Among the well-fitting Robitaille SED models are also configurations without a compact disk (axisymmetric flattened envelope + outflow cavities only), which nevertheless give almost identical elevated visibilities (see Fig. 5). This suggests that in the case of M8E IR, the choice of the central object actually governs the resulting visibility levels (see below).

We have also compared the spectral shape of the visibility curves with the models. For the comparison, we have computed synthetic images based on the parameters from the SED fits at eleven wavelengths between 8 and 13 micron. Their Fourier transform is then probed at spatial frequencies according to the projected baseline lengths of the MIDI measurements. In general, the Robitaille models predict somewhat lower visibilities than indicated by the measurements. The general shape of all the synthetic visibility curves is similar. Therefore, we show as one example the 46.8-m baseline comparison in Fig. 4. We compare the best envelope + disk configuration (Robitaille model 3007526, see Table 2) and the best envelope-only configuration (Robitaille model 3004120), both having a cool central object (see also Fig. 5), to the MIDI measurement. The model visibility curves show a clear decrease in the visibility level toward the amorphous silicate feature. The modelled shape would arise naturally in the circumstellar disk context when the correlated flux comes mainly from an inner, optically thicker region with a somewhat suppressed silicate emission feature, while the total flux is dominated by more optically thin emission from the wider disk surface (van Boekel et al. 2005). However, as seen in Fig. 4, the envelope-only model also gives the same behaviour. Hence, based on the modelled visibilities, it is not possible to distinguish between the two cases for our special setup. This is understandable considering the compactness of the disk in question, which would not be resolved even by our longest baseline measurement. The envelope by far dominates the mid-infrared emission of the whole object. The measured visibilities also show a level decrease. However, it is not centered around 9.7 micron, but occurs at longer wavelengths. Currently, we do not understand this shift. It is not clear if it can be fully explained by differing dust properties in M8E-IR and assumed for the radiative transfer simulations (opacities and grain size model from Laor \& Draine 1993; Kim et al. 1994). To further analyse and reproduce the spectral shape details of the visibility curves is beyond the scope of this paper.

We explicitly mention the low inclination angle of $<30^{\circ}$ inherent in all our Robitaille models fitting the SED data. This is in contrast to the disk hypothesis of Simon et al. (1985) that featured a very large inclination. However, Simon et al. saw a more or less symmetric intensity distribution in their $10 \mu \mathrm{m} \mathrm{lu}-$ nar occultation data; hints for deviations came mainly from lunar occultations recorded at $3.8 \mu \mathrm{m}$. At this shorter wavelength, configurations with disk + envelope including outflow cones are naturally more structured, and scattered light in the inner regions of these cones can still contribute, in particular if grains larger than the typical $0.1 \mu \mathrm{m}$ dust particles are involved.

\subsubsection{Comparison with canonical 1D models}

As mentioned above, the Robitaille models are not optimally sampled in the parameter ranges appropriate for massive YSOs. Formally, the Robitaille models cover a sufficiently large
Comparison MIDI vs models (46.8 m baseline)

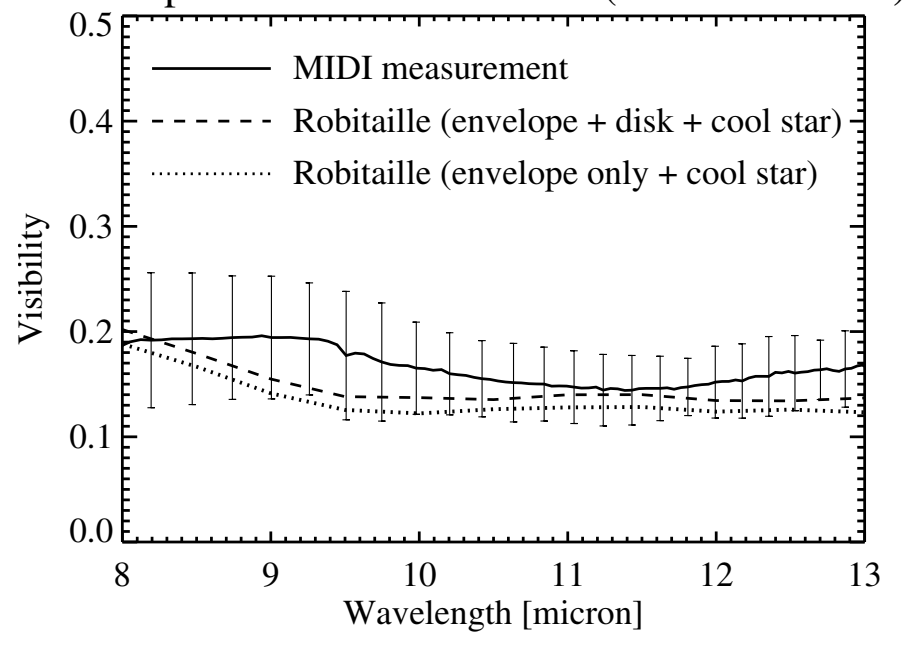

Fig. 4. Spectral shape comparison of the visibilities for the 46.8-m baseline as an example. The MIDI measurement plus 3- $\sigma$ error bars is given as a solid line. Furthermore, radiative transfer synthetic images between 8 and 13 micron have been produced for the best envelope + disk model (dashed line) and the best envelope-only model (dotted line). Cuts through their spatial frequency spectrum corresponding to the $46.8-\mathrm{m}$ baseline are concatenated to display the synthetic spectral visibility shape of these models.

parameter space regarding the 14 important parameters. In particular, hot, non-bloated central stars are included, up to effective temperatures of $>50000 \mathrm{~K}$ (see Fig. 3 in Robitaille et al. 2006). However, not all parameter combinations are available for the fitting since it is not possible to explore the huge parameter space in its entirety in a completely unbiased manner when constructing the model grid. Hence, the set of models available for the SED fitting does not form a complete "grid" in a technical sense, since parameters are randomly sampled within ranges (cf. Robitaille et al. 2006, especially Sects. 2.1.4 and 2.2.1). Since we have found quite peculiar models from our fitting attempt (see above), we felt compelled to do a cross-check of our results. We could have missed simpler models among the top-rank fitting results that nevertheless agree with the observed SED. Traditionally, such BN-type objects have been modelled as a spherical dust shell surrounding a hot, non-bloated central star (e.g., Gürtler et al. 1991; de Wit et al. 2009). To check if even such canonical configurations can account for the SED and the visibilities, we employed well-tested models, based on the code used by Men'shchikov \& Henning (1997); Men'shchikov et al. (1999). Unlike the Whitney code, the Men'shchikov program is a grid-based radiative transfer code. It comes with its own ray-tracing module to produce high-definition intensity maps. Here, we can tune the parameters without being restricted by a predefined parameter grid, and the fine-tuning process in the case of pure 1D models can be much faster. With a spherically symmetric envelope (density power law $\sim r^{-1.7}, M_{\mathrm{env}}=40 M_{\odot}$ ) and a $30000 \mathrm{~K}$ central star with $3.7 R_{\odot}$, it is possible to fit the SED of M8E-IR well (Fig. 3, see also de Wit et al. 2009²). Still, compared to the measured visibilities, these models result

\footnotetext{
2 These authors find slightly different model parameters, because they try to find a balance between fitting the $24.5 \mu \mathrm{m}$ intensity profile and properly fitting the SED. Thereby, they take some of the existing single-dish (sub-)millimeter points as face value. There are indications, however, that at long wavelengths the neighbouring UCHII region (Fig. 1, left) dominates the emission of the region. Therefore, these long-wavelength fluxes should be considered as strict upper limits.
} 
RT models vs observations for M8E-IR at 12 micron

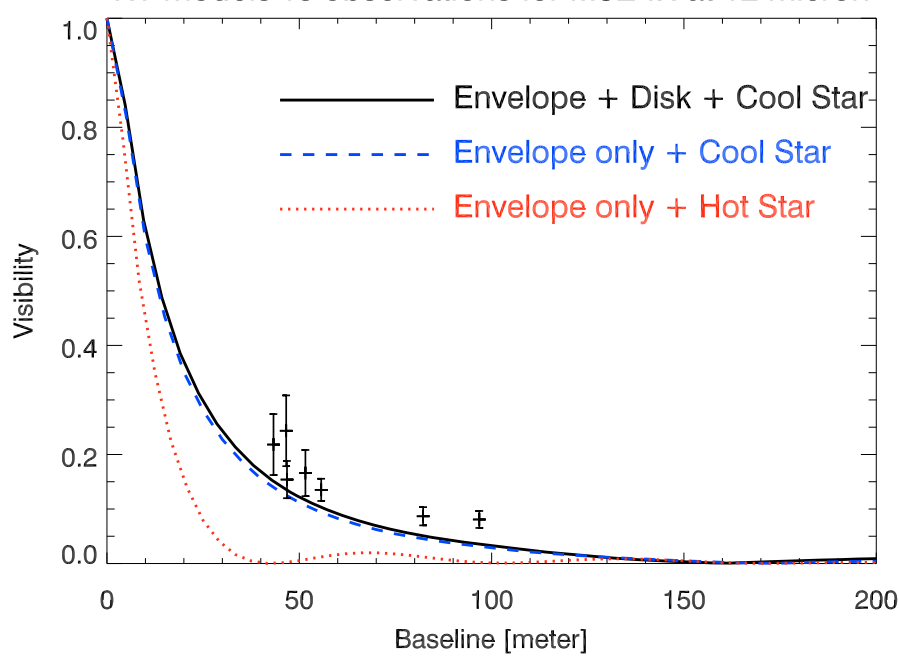

Fig. 5. Cuts through the $12 \mu \mathrm{m}$ spatial frequency spectrum (in units of the interferometric baseline length) of 3 models well fitting the SED. The black solid line refers to a model with a small circumstellar disk, flattened envelope and cool bloated central object (Table 2). The blue dashed line denotes a model with a flattened envelope and cool bloated central object without a disk; the intensity distribution is almost identical to the first model. Due to the low inclination of these 2D models $\left(18^{\circ}\right)$, the central intensity distribution shows only small deviations from radial symmetry. Hence, differences in the model visibilities with varying position angle remain small $(<10 \%)$, and the cuts can be adopted as representative. The classical approach (hot central star + spherical envelope, red dotted line), however, gives far too low visibilities compared to the MIDI data (plotted as plus signes including the formal $3 \sigma$ error bars).

in far too low visibilities $(<0.05)$ for M8E-IR in the baseline range 30-60 $\mathrm{m}$ over the whole $8-13 \mu \mathrm{m}$ range, even when we add a population of very large grains $(200 \mu \mathrm{m})$ to the central shells to raise the visibilities. The error margins of the MIDI visibilities (on average 10\%) do not account for such large differences. Independently, we found that the inclusion of a cold ( $2000 \mathrm{~K})$ central object will sufficiently raise the visibilities also in the Men'shchikov models. The circumstances are illustrated in Fig. 5 where the $(u, v)$-spectra of the $12 \mu$ m synthetic images based on 3 different models are compared. At such a longer wavelength outside the silicate absorption feature, the influence of different dust models on the results should be small. Furthermore, potential observational biases due to decreased beam overlap during the MIDI measurements are smaller at longer wavelengths (see Sect. 3.2). Although the (u,v)-spectra of the cool star models still show somewhat lower visibilities than the measured ones (the visibilities are not included in the fitting loop), obviously they are qualitatively different from the hot star model.

\subsubsection{Feasibility of a cool central object}

Interestingly, the best-fitting models for M8E-IR in the Robitaille model grid show central stars of $10-15 M_{\odot}$ that are strongly bloated and, therefore, have relatively low effective temperatures $(<5000 \mathrm{~K})$. Such solutions can occur since the Robitaille grid also comprises the full range of canonical pre-main sequence evolutionary tracks from the Geneva group ${ }^{3}$ as possible

\footnotetext{
3 http://obswww . unige.ch/Recherche/evol/ Geneva-grids-of-stellar-evolution
}

parameterisation of the central objects. Although M8E-IR cannot straightforwardly be identified with corresponding very early evolutionary stages, the tendency for a bloated central star in the case of M8E-IR may be valid. As found already by Kippenhahn \& Meyer-Hofmeister (1977), accretion with high rates onto main sequence stars can temporarily puff up such stars. Furthermore, Hosokawa \& Omukai (2008, 2009) and Yorke \& Bodenheimer (2008) have recently computed the pre-main-sequence evolution of stars as a function of the accretion rate onto the forming star. They find that for accretion rates reaching $10^{-3} M_{\odot} / y r$, the protostellar radius can temporarily increase to $>100 R_{\odot}$, in accordance with our model fitting. Note that Mitchell et al. (1988) revealed high-velocity molecular outflows from M8E-IR based on $M$-band $\mathrm{CO}$ absorption spectra and speculated on recent $(<120$ yr) FU Ori-type outbursts for this object. If these multiple outflow components really trace recent strong accretion events, the central star indeed could have been affected. Furthermore, M8E-IR is not detected by $\mathrm{cm}$ observations with medium sensitivity (Simon et al. 1984; Molinari et al. 1998). This could be explained by large accretion rates still quenching a forming hypercompact HII region (e.g., Walmsley 1995). Similarly, a bloated central star with $T_{\text {eff }} \ll 10000 \mathrm{~K}$ also gives a natural explanation of these findings.

\section{Conclusions}

We have observed the BN-type massive young stellar object M8E-IR with the MIDI MIR interferometer at the VLTI. We find substructures with MIR sizes around 30 mas. The measured elevated visibilities indicate that the usual approach for BN-type objects (a spherically symmetric envelope with a hot central star) fails in the case of M8E-IR. Most interestingly, our data are consistent with M8E-IR harbouring a $10-15 M_{\odot}$ central star that has been bloated $\left(R>100 R_{\odot}\right)$ by recent strong accretion events. However, with the present data we cannot clearly infer the existence of a circumstellar disk since disk-envelope and envelope-only models result in very similar SEDs and visibilities. If a disk exists it cannot be large ( $\$ 100$ AU). Until (sub-)millimeter observations with sufficient spatial resolution constrain the long-wavelength emission, disks with radii from 15-100 AU seem probable. Both aspects, the central star bloating and the existence of a circumstellar disk, should be the topic of future investigations. For instance, by utilising highresolution IR spectroscopy one can test whether hydrogen recombination lines show imprints of disk rotation along the guidelines of the disk-wind simulations by Sim et al. (2005).

Acknowledgements. Based in part on Subaru Telescope data and obtained from the SMOKA data archive, which is operated by the Astronomy Data Center at NAOJ. We thank Thomas Robitaille, Barbara Whitney, and Melvin Hoare for discussions on the subject. Valuable comments from the referee are appreciated.

\section{References}

Arias, J. I., Barbá, R. H., \& Morrell, N. I. 2007, MNRAS, 374, 1253

Baba, H., Yasuda, N., Ichikawa, S.-I., et al. 2002, in ASP Conf. Ser. 281, ed. D. A. Bohlender, D. Durand, \& T. H. Handley, 298

Beltrán, M. T., Brand, J., Cesaroni, R., et al. 2006, A\&A, 447, 221 Bjorkman, J. E., \& Wood, K. 2001, ApJ, 554, 615

Damiani, F., Prisinzano, L., Micela, G., \& Sciortino, S. 2006, A\&A, 459, 477 De Buizer, J. M. 2006, ApJ, 642, L57

de Wit, W.-J., Hoare, M. G., Oudmaijer, R. D., \& Mottram, J. C. 2007, ApJ, 671, L169

de Wit, W. J., Hoare, M. G., Fujiyoshi, T., et al. 2009, A\&A, 494, 157 Fujiwara, H., Honda, M., Kataza, H., et al. 2006, ApJ, 644, L133 Gürtler, J., Henning, T., Krügel, E., \& Chini, R. 1991, A\&A, 252, 801 Henning, T. 1990, Fundamentals of Cosmic Physics, 14, 321 
Hosokawa, T., \& Omukai, K. 2008, in ASP Conf. Ser. 387, ed. H. Beuther, H. Linz, \& T. Henning, 255

Hosokawa, T., \& Omukai, K. 2009, ApJ, 691, 823

Kim, S.-H., Martin, P. G., \& Hendry, P. D. 1994, ApJ, 422, 164

Kippenhahn, R., \& Meyer-Hofmeister, E. 1977, A\&A, 54, 539

Laor, A., \& Draine, B. T. 1993, ApJ, 402, 441

Leinert, C., Graser, U., Waters, L. B. F. M., et al. 2003, in Interferometry for Optical Astronomy II, ed. W. A. Traub, Proc. SPIE, 4838, 893

Leinert, C., van Boekel, R., Waters, L. B. F. M., et al. 2004, A\&A, 423, 537

Linz, H., Stecklum, B., Henning, T., Hofner, P., \& Brandl, B. 2005, A\&A, 429, 903

Men'shchikov, A. B., \& Henning, T. 1997, A\&A, 318, 879

Men'shchikov, A. B., Henning, T., \& Fischer, O. 1999, ApJ, 519, 257

Mitchell, G. F., Allen, M., Beer, R., et al. 1988, ApJ, 327, L17

Mitchell, G. F., Hasegawa, T. I., \& Schella, J. 1992, ApJ, 386, 604

Molinari, S., Brand, J., Cesaroni, R., Palla, F., \& Palumbo, G. G. C. 1998, A\&A, 336,339

Mueller, K. E., Shirley, Y. L., Evans, II, N. J., \& Jacobson, H. R. 2002, ApJS, 143,469

Okamoto, Y. K., Kataza, H., Yamashita, T., et al. 2003, in Proc. SPIE 4841, ed. M. Iye, \& A. F. M. Moorwood, 169
Prisinzano, L., Damiani, F., Micela, G., \& Sciortino, S. 2005, A\&A, 430, 941 Robitaille, T. P. 2008, in ASP Conf. Ser. 387, ed. H. Beuther, H. Linz, \& T. Henning, 290

Robitaille, T. P., Whitney, B. A., Indebetouw, R., Wood, K., \& Denzmore, P. 2006, ApJS, 167, 256

Robitaille, T. P., Whitney, B. A., Indebetouw, R., \& Wood, K. 2007, ApJS, 169, 328

Sim, S. A., Drew, J. E., \& Long, K. S. 2005, MNRAS, 363, 615

Simon, M., Cassar, L., Felli, M., et al. 1984, ApJ, 278, 170

Simon, M., Peterson, D. M., Longmore, A. J., Storey, J. W. V., \& Tokunaga, A. T. 1985, ApJ, 298, 328

Val'tts, I. E. 1999, Astron. Rep., 43, 149

van Boekel, R., Dullemond, C. P., \& Dominik, C. 2005, A\&A, 441, 563

Vehoff, S., Nürnberger, D. E. A., Hummel, C. A., \& Duschl, W. J. 2008, in ASP Conf. Ser. 387, ed. H. Beuther, H. Linz, \& T. Henning, 444

Walmsley, M. 1995, in Rev. Mex. Astron. Astrofis. Conf. Ser. 1, ed. S. Lizano, \& J. M. Torrelles, 137

Whitney, B. A., Wood, K., Bjorkman, J. E., \& Wolff, M. J. 2003, ApJ, 591, 1049 Yorke, H. W., \& Bodenheimer, P. 2008, in ASP Conf. Ser. 387, ed. H. Beuther, H. Linz, \& T. Henning, 189 